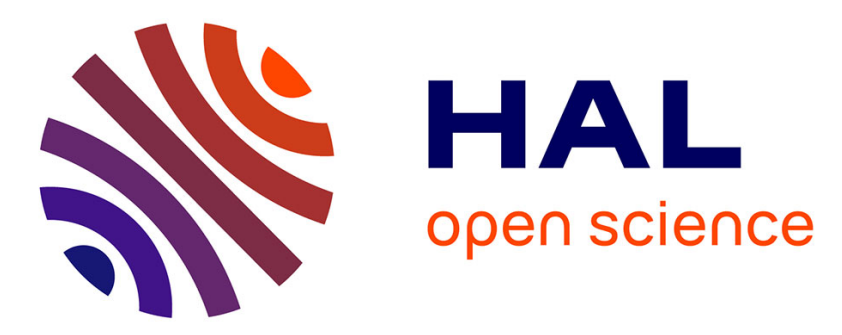

\title{
Acoustical power computation acceleration techniques for the planning of ultrasound therapy
}

Jean-Louis Dillenseger, Carole Garnier

\section{To cite this version:}

Jean-Louis Dillenseger, Carole Garnier. Acoustical power computation acceleration techniques for the planning of ultrasound therapy. 5th IEEE International Symposium on Biomedical Imaging ISBI'08, May 2008, Paris, France. pp.1203-1206. inserm-00280797

\section{HAL Id: inserm-00280797 https://www.hal.inserm.fr/inserm-00280797}

Submitted on 19 May 2008

HAL is a multi-disciplinary open access archive for the deposit and dissemination of scientific research documents, whether they are published or not. The documents may come from teaching and research institutions in France or abroad, or from public or private research centers.
L'archive ouverte pluridisciplinaire HAL, est destinée au dépôt et à la diffusion de documents scientifiques de niveau recherche, publiés ou non, émanant des établissements d'enseignement et de recherche français ou étrangers, des laboratoires publics ou privés. 


\title{
ACOUSTICAL POWER COMPUTATION ACCELERATION TECHNIQUES FOR THE PLANNING OF ULTRASOUND THERAPY
}

\author{
J-L. Dillenseger, C. Garnier \\ INSERM, U642, Rennes, F-35000, France \\ Université de Rennes 1, LTSI, Rennes, F-35000, France
}

\begin{abstract}
The simulation of the effect of a high intensity ultrasound interstitial therapy is mainly connected on an accurate estimation of the pressure delivered by the transducer. This paper describes and evaluates several simplifications methods used to estimate this pressure field and the induced acoustical power.
\end{abstract}

\section{INTRODUCTION}

Interstitial ultrasound applicators are now proposed for the curative treatment of hepatocellular carcinoma (HCC) [1]. Interstitial ultrasound offers the advantage to be more controllable in depth and direction than other interstitial heating modalities. Like any other minimally invasive image guided therapy, its clinical application requires an efficient planning at the pre-operative stage. Thus, in addition to the classical image processing issues (segmentation, registration, rendering, etc.), a physically-based model of the thermal dose to be delivered must be designed. Classically in hyperthermia, the tissue temperature $T$ evolution over time (and so the estimation of the necrotic volume) is obtained by solving Pennes' bioheat transfer equation (BHTE) [2] which can be summarized by: $\rho C \frac{\partial T}{\partial t}=k \nabla^{2} T+h_{b}+Q$ where $\rho$ and $C$ refer to tissue density and specific heat, $k$ the thermal conductivity of tissue, $h_{b}$ the rate of heat transfer per unit volume of tissue induced by the blood perfusion and $Q$ the rate of the heat per unit volume of tissue produced by the source. In the case of ultrasound therapy, $Q$ is an acoustical power given by: $Q=\mu_{0} f p^{2} / \rho c$ where $\mu_{0}$ is the absorbtion coefficient of ultrasound in tissues; $f$, the ultrasound frequency; $c$, the ultrasound velocity and $p$, the pressure delivered by the ultrasound device. The estimation of an accurate pressure field $p$ is one of the key points of the simulation. Under some linearity assumptions the pressure field can be exactly estimated on the basis of the Rayleigh integral. However, the numerical resolution of the Rayleigh integral is very computation intensive. This computation can be simplified by help of some assumptions about tissue spatial homogeneity and invariance

This work is part of the national SUTI project supported by an ANR Grant (ANR05RNTS01106). of its acoustic properties. In this paper we will examine these assumptions and evaluate their impact on computation speed and accuracy of the estimated pressure field.

\section{METHODS}

\subsection{Ultrasound device and principle of the acoustic pres- sure computation}

The modeled therapeutic ultrasound device (Fig. 1) was composed of a small $(3 \mathrm{~mm} \times 9 \mathrm{~mm})$ planar ultrasonic monoelement transducer encapsulated in a $\varnothing 4 \mathrm{~mm}$ cylindrical interstitial applicator. The transducer was air-backed, so ultrasound was only propagated forward. The front transducer face was cooled by a continuous flow of degassed water maintained at a constant temperature.

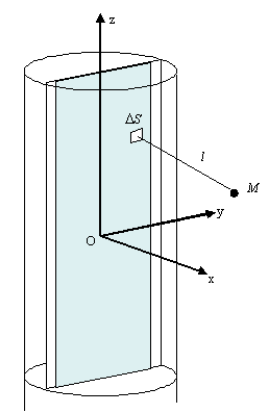

Fig. 1. Ultrasound device geometry and coordinates system.

The goal of the work is to compute the acoustic pressure at the location points $M$ sampled in the volume in a cartesian or cylindrical coordinates system.

In our study, with a non-focused plane transducer, the propagation can be considered as linear. The pressure field can be exactly computed for the entire volume on the basis of the Rayleigh integral with O'Neil's hypotheses [3]. The transducer of surface $S$ is sampled into elements of surface $\Delta S$ which size is negligible in comparison with the wavelength $\lambda ; M$ and one element of surface $\Delta S$ are connected by a straight segment $\Delta S M$ of length $l$ sampled into elements $\Delta l$. The pressure at a location $M$ is given by the discrete 
version of the Rayleigh integral:

$$
p(M)=\left|\sum_{S} j \frac{p_{0}}{\lambda} \Delta S \frac{\exp ^{-j k l}}{l} \exp ^{-f \sum_{i=1}^{l} \alpha_{i} \Delta l}\right|
$$

with $p_{0}$ the pressure at the transducer surface $(\mathrm{Pa}), k$ the wave number $(2 \pi / \lambda), f$ the transducer frequency $(\mathrm{Hz})$ and $\alpha_{i}$ the tissue attenuation coefficient at location $i$ along the line segment $\triangle S M\left(\mathrm{~Np} \cdot \mathrm{m}^{-1} \cdot \mathrm{MHz}^{-1}\right)$.

This twofold integration (over the surface of the transducer and the segment $\Delta S M$ ) is very computation intensive. Some simplifications are usually made in order to accelerate the simulation.

\subsection{Homogeneous tissue}

The tissues are assumed to be homogeneous around the applicator and its physical properties (the tissue attenuation coefficient) constant in time. The volume is thus composed by 2 elements: the cooling water of the transducer and the tissue with respectively an attenuation coefficient of $\alpha_{1}$ and $\alpha_{2}$.

The simulation can be accelerated using:

1. Symmetry. The simulated air-backed transducer providing only forward waves and the homogeneous medium of propagation allow computing pressure in a quarter of a space in front of the propagation axis only for symmetry reasons.

2. Rayleigh integral simplification. The propagation of a wave from one element of surface $\Delta S$ to $M$ is carried through only 2 media: the cooling water of the transducer $\left(\alpha_{1}, l_{1}\right)$ and the tissues $\left(\alpha_{2}, l_{2}\right)$. Because $\alpha_{1}$ is negligible and $p_{0}$ is estimated at the surface of the applicator, Eq. 1 can therefore be simplified as:

$$
p(M)=\left|\sum_{S} j \frac{p_{0}}{\lambda} \Delta S \frac{\exp ^{-j k l}}{l} \exp ^{-f \alpha_{2} l_{2}}\right|
$$

3. Transducer sampling. The size of $\Delta S$ must be negligible in comparison with the wavelength $\lambda$. A size of $\Delta S$ between $\lambda / 10$ and $\lambda / 5$ is currently admitted. A large size of $\Delta S$ would permit to speed up the computation but a good compromise between the large size of $\Delta S$ and the calculation accuracy must be found.

4. Adaptative volume sampling. The pressure variation is not constant over the volume. The regions near the transducer, presents large pressure variation in the contrary of the more distant regions where the pressure is almost constant. We used an adaptative sampling of the points $M$ over the volume. The pressure is first computed on one sample point every 8 sample points on each direction. If the pressure values computed on adjacent sampled points is bellow than a first threshold $P_{1}=\max (p) / 2$ or if the difference between those pressure values is less than a second threshold $P_{2}=$ $\max (p) / 100$, the pressure on the intermediate sample point is computed by interpolation, otherwise by Eq. 2 .

5. Rayleigh integral approximation. In an early work, Freedman [4] made some approximations on the $\int_{S} \frac{\exp ^{-j k l}}{l}$ phase term of the integrant of Eq. 2: 1) the amplitude term $1 / l$ is approximated by $1 / L$ where $L$ is the distance between $M$ and the center $\mathrm{O}$ of the transducer; 2 ) the distance $l$ in $\exp ^{-j k l}$ is expanded binomially and the first two terms are kept. This later approximation allows to formulated the integration over $S$ in the form of complex Fresnel integrals which can be easily estimated:

$p_{n}(M)=\int_{S} \frac{\exp ^{-j k l}}{l} \approx \frac{x}{2 L} \exp ^{-j k x}(\mathbf{F}[\beta(y+a)]-$ $\mathbf{F}[\beta(y-a)])(\mathbf{F}[\beta(z+b)]-\mathbf{F}[\beta(z-b)])$, with $a$ and $b$ the half width of the transducer in direction $y$ and $z$; $\beta=\sqrt{k / \pi x}$ and $\mathbf{F}[\xi]=\int_{0}^{\xi} \exp ^{j \pi \zeta^{2} / 2} d \zeta$, the complex Fresnel integral which can be evaluated using fast rational approximation or pre-computed on a look-uptable. More recently Mast et al. [5] add an attenuation term but only between $M$ and the center $\mathrm{O}$ of the transducer:

$$
p(M)=\left|\frac{p_{0}}{\lambda} p_{n}(M) \exp ^{-f \alpha_{2} L_{2}}\right|
$$

where $L_{2}=L-$ applicator radius. However, these approximations are only valid on a certain distance from the transducer. Freedman [4] found the minimum axial range: $x_{\text {min }}=b \sqrt{1+(a / b)^{2}} / \tan 32^{\circ} \approx 15 \mathrm{~mm}$.

\subsection{Dynamic tissue attenuation and absorption coeffi- cients variation}

The absorption $\alpha$ and attenuation $\mu_{0}$ coefficients associated to a given tissue change as temperature changes and as tissue necroses (usually these both coefficients are supposed to be very close, on the rest of the paper this coefficients will be considered as equal). The attenuation coefficient is no more constant over the volume. The attenuation coefficient curves (ie coefficient variation vs. temperature) was measured for various tissues [6]. For liver, Connor et al. [7] made a 6th order polynomial approximation of the experimental data (see curve in Fig. 2).

It has been shown through simulation that the shape and size of the resulting necrotic volume is significantly different when this variation is taken into account from the one obtained when this tissue property is considered constant [8].

This variation of $\alpha$ and $\mu_{0}$ will have two impact on the simulation because: 1) the acoustical power $Q$ is directly proportional to $\mu_{0}$, and 2) the pression estimation is dependant to $\alpha$ 


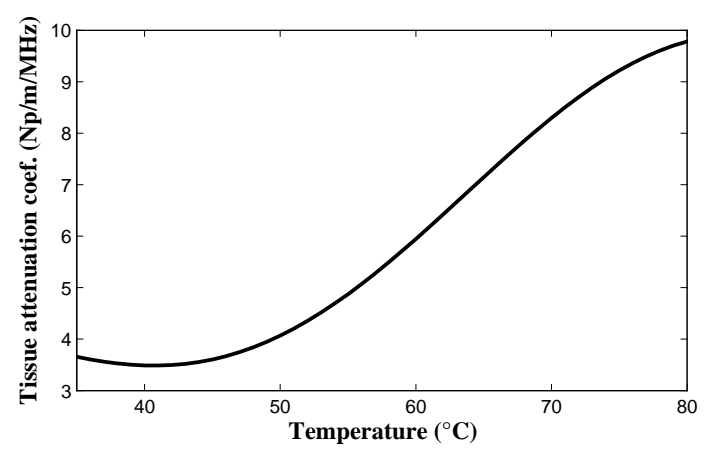

Fig. 2. Linearized tissue attenuation coefficient vs. temperature.

The first point can be easily integrated in the computation framework of $Q=\mu_{0} f p^{2} / \rho c$. At a time step, the BHTE gives the temperature on each point $M$. The absorbtion coefficient $\mu_{0}$ can then been directly estimated from the curve shown in Fig. 2.

Considering the second point, because the hypothesis of homogeneity of the tissue properties is no more true, some previous described simplifications of the estimation of $p$ (\#1symmetry, \#2-Rayleigh integration simplification and \#5Ray- leigh integral approximation) cannot be used anymore or must be adapted. The reference algorithm based on Eq. 1 induces a tremendous computing time. However, several new simplifications can be proposed in order to reasonably reduce this computing time without loosing accuracy:

6. Segments coherence. Adjacent $\Delta S M$ segments share almost the same attenuation properties. For nine adjacent connected transducer surface element $\Delta S$, we compute the $\exp ^{-f \sum_{i=1}^{l} \alpha_{i} \Delta l}$ term of Eq. 1 for only the $\Delta S M$ segment which originates from the central surface element and apply this term to the segments which originate from the eight adjacent surface elements.

7. Attenuation approximation. Mast et al. [5] extend the previous idea and apply only the attenuation term computed between $M$ and the center $\mathrm{O}$ of the transducer. This attenuation term can so be multiplied outside of the integrant.

\section{RESULTS}

We simulated the pressure field $p$ and the power deposit $Q$ induced by a $5 \mathrm{MHz}$ transducer with a acoustical intensity of $40 \mathrm{~W} / \mathrm{cm}^{2}$. The volume was regularly sampled on $191 \times 100$ $\mathrm{x} 121$ points.

\subsection{Homogeneous tissue}

The volume computed with a transducer sampled by $\Delta S$ with a size of $\lambda / 10$ will be considered as the reference. Several
Table 1. Pressure field estimation errors and computation time vs. simplification methods

\begin{tabular}{|r|c|c|c|c|c|}
\hline & $\begin{array}{c}\text { mean abs } \\
\text { error }\end{array}$ & $\begin{array}{c}\text { mean rel } \\
\text { error \% }\end{array}$ & $\begin{array}{c}\text { RMS } \\
\text { error }\end{array}$ & $\begin{array}{c}\text { comp. } \\
\text { time (mn) }\end{array}$ & $\begin{array}{c}\text { acc. } \\
\text { rate }\end{array}$ \\
\hline$\lambda / 10$ & 0 & 0 & 0 & 60.5 & \\
\hline$\lambda / 8$ & 160 & 0.1 & 265 & 38.9 & 1.6 \\
\hline$\lambda / 6$ & 511 & 0.32 & 845 & 21.8 & 2.8 \\
\hline$\lambda / 5$ & 869 & 0.54 & 1439 & 15.2 & 4 \\
\hline$\lambda / 4$ & 1543 & 0.96 & 2564 & 9.7 & 6.2 \\
\hline$\lambda / 2$ & 8047 & 5.1 & 13802 & 2.4 & 25 \\
\hline \hline$\# 4$ & 1705 & 1.2 & 4928 & 4.4 & 13.75 \\
\hline \hline$\# 5$ & 59473 & 23 & 108494 & $0.29(\mathrm{~s})$ & 12500 \\
\hline \hline$\# 6$ & 0 & 0 & 0 & 38 & \\
\hline$\# 7$ & 30276 & 15 & 42516 & 5.3 & 7.2 \\
\hline
\end{tabular}

measurements have been performed to compare the pressure field obtained by the several simplification methods to the reference one: mean absolute error, mean relative error, RMS error, computation time and acceleration rate (computation time of the volume computed with $\lambda / 10$ divided by computation time of the volume computed with the accelerated method).

Effect of the transducer sampling (simplification \#3). In Eq. 2, the size of $\Delta S$ is increased from $\lambda / 10$ to $\lambda / 2$. The results summarized on Table 1 shows that until $\lambda / 5$, the errors remain relatively low with a real gain in computation time. For the rest of the paper, a $\Delta S$ size of $\lambda / 5$ will be considered.

Adaptative volume sampling (simplification \#4). Beyond the several tests we made, we retains the case where $\Delta S$ had a size of $\lambda / 5$. In this case, the pressure value was only computed on around $30 \%$ of the volume sampling points. The errors are still reasonable with a good computation speed-up (see the line labeled \#4 on Table 1).

Rayleigh integral approximation (simplification \#5). This method is very fast (less than a second) but with very high errors (see see the line labeled \#5 on Table 1).

\subsection{Dynamic tissue attenuation and absorption coeffi- cients variation}

In order to evaluate the impact of $\alpha$ and $\mu_{0}$ within the estimation of $p$ and $Q$, we take a temperature map computed by simulation from a previous work [8]. Using the curve shown in Fig. 2, we estimated the coefficients $\alpha$ and $\mu_{0}$ for each sampling points $M$.

Influence of $\alpha$ for the pressure field estimation. We simulated the pressure field using the two methods described previously: simplifications \#6 (segments coherence) and simplification \#7 (attenuation approximation). In order to decrease 
the computation time, the pressure fields were computed by adaptative volume sampling with a $\Delta S$ size of $\lambda / 5$. Fig. 3 shows the profile of the pressure field along the $\mathrm{x}$ axis $(\mathrm{y}=0$, $\mathrm{z}=0$, see Fig. 1 for the coordinates system definition). The profiles are notably different when the absorption coefficients variation is taken (labels: Segment coherence and Att approximation) or not (label: Constant alpha) into account. Comparing now the two simplifications methods, even if their profiles seems to be close, some differences exist between these two methods (see Table 1, lines labeled \#6 and \#7; the simplification method \#6 was considered as reference for the errors and the acceleration rate). If we have a closer look to the locations of these errors, they mostly happen near the applicator on a distance less than $10 \mathrm{~mm}$ to its center.

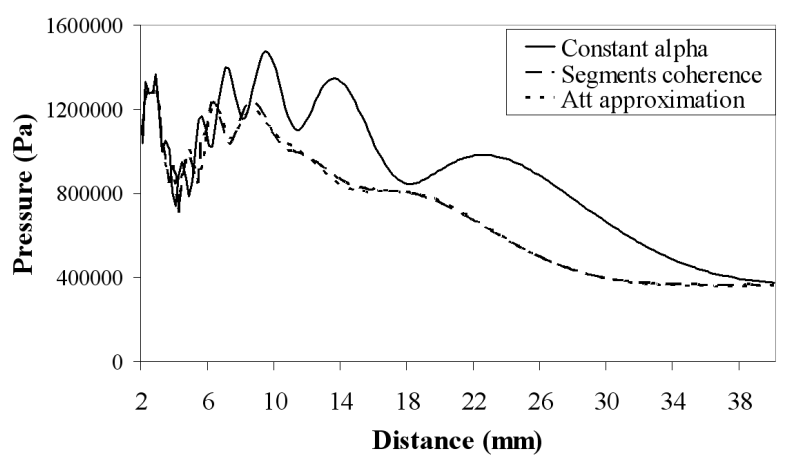

Fig. 3. Pressure along the axial direction.

Influence of $\mu_{0}$ for the acoustical power estimation. Fig. 4 shows the profile of the acoustical power field along the $\mathrm{x}$ axis. Three curves are represented: the field estimated with: 1) constant $\mu_{0}$ and $\alpha$ (label: mu constant); 2) the variation of $\mu_{0}$ but with constant $\alpha$ (label: $m u$ varying, alpha constant) and 3) the variation of both $\mu_{0}$ and $\alpha$ (label: mu and alpha varying). The profiles are notably different depending on the complexity of the model.

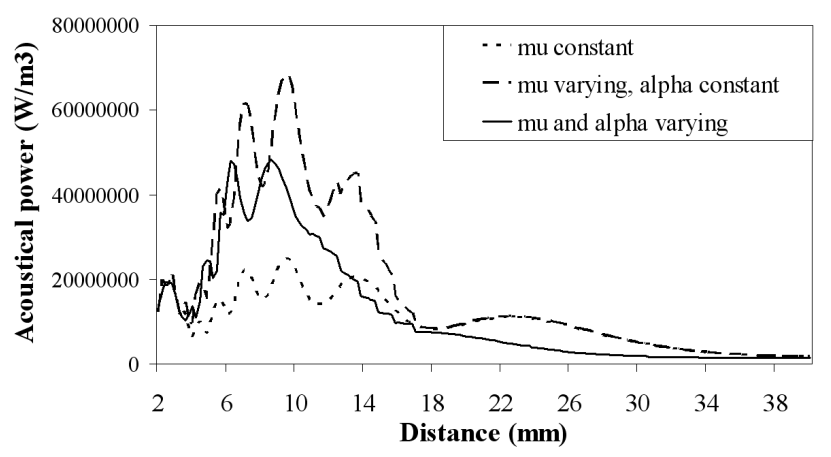

Fig. 4. Acoustical power along the axial direction.

\section{DISCUSSION AND CONCLUSION}

The previous results demonstrate that the level of realism of the models has a direct impact on the simulated pressure field and acoustical power deposit but to the detriment of computation time. However, some simplifications lead to faster computation, but with higher errors. Further studies have to be performed in order to evaluate the impact of these errors on the temperature estimation. We expect that these errors on the acoustical power deposit could have only a small impact on the BHTE. Experimental validation should also be performed to validate the models.

\section{REFERENCES}

[1] C. Lafon, J. Y. Chapelon, F. Prat, F. Gorry, J. Margonari, Y. Theillere, and D. Cathignol, "Design and preliminary results of an ultrasound applicator for interstitial thermal coagulation," Ultrasound Med Biol, vol. 24, no. 1, pp. 113-22, 1998

[2] H. H. Pennes, "Analysis of tissue and arterial blood temperatures in the resting human forearm. 1948," J Appl Physiol, vol. 85, no. 1, pp. 5-34, 1998.

[3] C. Lafon, F. Prat, J. Y. Chapelon, F. Gorry, J. Margonari, Y. Theillere, and D. Cathignol, "Cylindrical thermal coagulation necrosis using an interstitial applicator with a plane ultrasonic transducer: in vitro and in vivo experiments versus computer simulations," Int J Hyperthermia, vol. 16, no. 6, pp. 508-22, 2000.

[4] A. Freedman, "Sound field of a rectangular piston," $J$ Acoust Soc Am, vol. 32, no. 2, pp. 197-209, 1960.

[5] T. D. Mast, I. R. Makin, W. Faidi, M. M. Runk, P. G. Barthe, and M. H. Slayton, "Bulk ablation of soft tissue with intense ultrasound: modeling and experiments," $J$ Acoust Soc Am, vol. 118, no. 4, pp. 2715-24, 2005.

[6] C. A. Damianou, N. T. Sanghvi, F. J. Fry, and R. MaassMoreno, "Dependence of ultrasonic attenuation and absorption in dog soft tissues on temperature and thermal dose," J Acoust Soc Am, vol. 102, no. 1, pp. 628-34, 1997.

[7] C. W. Connor and K. Hynynen, "Bio-acoustic thermal lensing and nonlinear propagation in focused ultrasound surgery using large focal spots: a parametric study," Phys Med Biol, vol. 47, no. 11, pp. 1911-28, 2002.

[8] C. Garnier, C. Lafon, and J.-L. Dillenseger, "3D modeling of the thermal coagulation necrosis induced by an interstitial ultrasonic transducer," IEEE Trans Biomed Eng, vol. 55, no. 2, pp. 833-7, 2008. 